EOMmUn: Communication et organisation

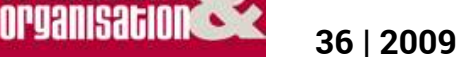

Pour une approche communicationnelle de l'individu au travail

\title{
L'activité du bibliothécaire entre ombre et lumière
}

Les formes plurielles de la reconnaissance

\section{Marie-France Peyrelong}

\section{(2) OpenEdition}

\section{Journals}

Édition électronique

URL : http://journals.openedition.org/communicationorganisation/933

DOI : 10.4000/communicationorganisation.933

ISSN : $1775-3546$

Éditeur

Presses universitaires de Bordeaux

Édition imprimée

Date de publication : 1 décembre 2009

Pagination : 82-94

ISBN : 978-2-86781-719-9

ISSN : 1168-5549

Référence électronique

Marie-France Peyrelong, « L'activité du bibliothécaire entre ombre et lumière », Communication et organisation [En ligne], 36 | 2009, mis en ligne le 02 mai 2011, consulté le 19 avril 2019. URL : http:// journals.openedition.org/communicationorganisation/933; DOI : 10.4000/

communicationorganisation.933 
Dossier : Pour une approche communicationnelle de l'individu au travail

\title{
Résumé
}

Comment l'activité de travail est-elle prise en considération par le management? Quelle attention porte-t-on à l'autre en situation « ordinaire » de travail ? Une tension entre visibilité et invisibilité est alors en jeu pour gagner ou donner de la reconnaissance. Ces questions sont adressées ici à partir d'une enquête auprès des cadres intermédiaires dans les bibliothèques sur la manière dont ils perçoivent les manifestations de reconnaissance sur le lieu de travail. Les premiers résultats mettent en évidence les formes plurielles que prend la reconnaissance selon qu'il s'agisse de mettre en visibilité son travail ou celui de son équipe.

\section{Mots clés}

Reconnaissance, visibilité, communication, cadres intermédiaires

\begin{abstract}
How does one's work get to be taken into consideration by the management particularly in daily, "ordinary" work situation? A whole tension of visibility and invisibility is at stake in order to gain or to give recognition. This issue will be addressed through an survey on what librarians who are in a middle management position say about recognition in their workplace. The first results show a plurality of forms of recognition whether the librarian has to shed the light on his own work or the work done by his team.
\end{abstract}

\section{Keywords}

Acknowledgement, visibility, communication, middle management

Marie-France Peyrelong est maître de conférences à l'Ecole Nationale Supérieure en Sciences de l'Information et des Bibliothèques (ENSSIB). Membre du Laboratoire ELICO et coordinatrice de l'axe "Communication et Organisations». Ses recherches portent sur les médiations en œuvre dans les partages et la circulation de l'information et des connaissances, le travail collaboratif, l'oral et l'écrit dans les organisations. 


\section{L'activité du bibliothécaire entre ombre et lumière. Les formes plurielles de la reconnaissance Marie-France Peyrelong}

marie-france.peyrelong@enssib.fr

Les questions relatives à la considération de l'individu au travail reviennent en force depuis le début des années 2000 avec les dénonciations des souffrances au travail (Dejours, 1998, 2003, 2005). Considérer, si l'on reprend des définitions communes, c'est regarder avec attention, en quelque sorte donner ainsi existence à autrui, remarquer sa singularité, son individualité et la qualifier positivement de digne d'intérêt. Quel regard porte-t-on sur l'autre ? Quelle attention porte-t-on à l'autre, non pas lorsqu'il s'agit d'un événement remarquable, publicisé sur l'intranet voire par des médias extérieurs à l'organisme, mais en situation ordinaire de travail? Comment l'individu est-il vu ou perçu dans la réalisation quotidienne du travail?

De nombreux instruments mis en place par le management participent de la mise en visibilité des individus au travail : rapport d'activité, organigramme, entretien d'évaluation, fiche de poste pour n'en citer que quelques-uns. Les formes managériales actuelles s'appuient fortement, et de plus en plus, sur une production scripturaire sur/de l'activité des individus au travail. En effet, comme le souligne Olivier Voirol (2005: 11), «la visibilité des activités se construit (...) à travers des dispositifs de formalisation du travail que constituent les procédés d'inscription textuelle ou graphique ». Ces documents, inscriptions de l'activité qui sont des intermédiations «jamais complètes [et qui peuvent] déboucher sur des illusions de vérité et d'objectivité » (Dupont, Dutrel, 2006 : 128), sont dotés d'un pouvoir performatif puisque, si on s'en tient aux exemples donnés, ils assignent une place, ou encore vont justifier une augmentation (ou non) de salaire ou une promotion (ou non) de l'individu. L'activité est donc mise en forme, fait l'objet de reporting divers, mais qu'est-il « reconnu » de l'activité d'un individu, voire de l'individu lui-même?

Les sociologues du travail et les ergonomes ont mis en évidence combien l'activité réelle s'éloigne de l'activité prescrite, ne serait-ce que pour que la production puisse être menée à bien. Toute une tranche de l'activité échappe donc à la visibilité ou encore à la reconnaissance des directions ou des hiérarchies, étant entendu que la visibilité, étape nécessaire, ne se superpose pas à cette dernière notion 


\section{Dossier : Pour une approche communicationnelle de l'individu au travail}

(Honneth, 2005 ; Voirol, 2005). L'idée n'est bien évidemment pas de souscrire à une idéologie de la «transparence» de l'activité des individus et il serait naif de penser que les décideurs cherchent à tout savoir. La mise en place des TIC démontre combien de tris sont opérés sur les éléments de l'activité productive qui seront « remontés » aux directions, obéissants à d'autres logiques d'acteurs. Il s'agit ici de pointer ce qui de l'activité sera remarqué et contribuera à la reconnaissance de la personne employée. Quelles marques de reconnaissance seront utilisées dans une organisation pour montrer cette disposition vis-à-vis d'autrui ? En quelque sorte au lieu de la mise à plat de l'activité que l'on tente d'effectuer par différents investissements de forme (Thévenot, 1986), nous nous intéresserons au contraire à la mise en relief de l'activité singulière d'un individu enchâssée dans une organisation.

\section{Problématique et terrain}

C'est cette question de la visibilité de l'activité comme participant à la reconnaissance de l'individu au travail et du rôle central de la communication dans ce processus que nous souhaitons discuter dans cet article. Valérie Larroche (2008) a exposé l'ancrage de la reconnaissance comme concept opératoire dans le champ des SIC en ce qu'elle combine une dimension relationnelle et organisationnelle. Dans le cadre d'une recherche collective ${ }^{32}$ (Larroche, Peyrelong, Lépine, Laroux, 2008), nous avons fait l'hypothèse de la reconnaissance comme dispositif managérial et nous nous sommes intéressées à cette position particulière des cadres intermédiaires, à la fois relais de la direction, mais aussi des équipes.

Nous reprendrons ici l'un des deux terrains ${ }^{33}$ que nous avions commencé à explorer et qui concerne les bibliothécaires ${ }^{34}$. Le choix de terrains issus de la fonction publique permet de mettre entre

${ }^{32}$ Cette recherche fait l'objet d'une thématique de l'axe «Communication et Organisations » du laboratoire ELICO. Je voudrais ici remercier particulièrement $\mathrm{M}$. Follet et $\mathrm{O}$. Dupont pour leurs relectures et les discussions suscitées par cet article.

${ }^{33}$ Voir la contribution de V. Lépine sur les cadres intermédiaires de la santé dans ce même numéro.

${ }^{34} \mathrm{La}$ terminologie utilisée dans cet article est celle qui caractérise les différents corps et statuts existant dans les bibliothèques. Ainsi les personnels sont répartis en conservateurs et bibliothécaires (catégories A), bibliothécaires assistants spécialisés (catégorie B), magasiniers (catégorie C). Nos interlocuteurs travaillent dans des bibliothèques universitaires ou des bibliothèques de collectivités territoriales (municipales ou départementales). 
parenthèse le levier financier comme forme de reconnaissance, souvent prépondérante, dans le secteur privé.

Nous réinterrogerons ce corpus ${ }^{35}$ à la lueur de cette tension entre ce qui reste dans l'ombre et ce qui est mis en lumière, autour d'une activité qui est le plus souvent ressentie dans la profession comme invisible de l'extérieur. Comme le souligne Olivier Voirol, une «part considérable de l'expérience sociale consiste à transformer le spectre de la visibilité » (2005: 19), permettant à des groupes sociaux ou professionnels de pouvoir être pris en compte. Ce n'est cependant pas à cette dimension sociétale, politique que nous nous intéresserons ici, mais plutôt à l'expérience ramenée au niveau des individus pris/agissant dans des relations au sein d'un collectif et dans des organisations. C'est à partir de leur parole ${ }^{36}$, de leur perception, que nous tenterons de comprendre les manifestations de reconnaissance que ces bibliothécaires peuvent mettre en œuvre vis-à-vis de leur équipe et qu'ils peuvent à leur tour recevoir de leur hiérarchie.

Dans un premier temps, le contexte de cette activité d'encadrement intermédiaire que constitue le travail de bibliothécaire sera rappelé. Nous examinerons comment ensuite se met en scène la reconnaissance pour soi et pour autrui. Pour soi : quelles attentes le bibliothécaire a-til vis-à-vis de sa hiérarchie en matière de reconnaissance de son travail ? Comment perçoit-il la reconnaissance que sa hiérarchie lui attribue ? Quelles marques reçoit-il de cette dernière dans son activité de travail ? Pour autrui : comment à son tour, le bibliothécaire, en tant que responsable d'une équipe met-il en œuvre des marques de reconnaissance? Que met-il en visibilité ? Paroles singulières, « situées », c'est-à-dire propres à des places (Charasse, 1992) occupées et attribuées à un moment donné dans une organisation particulière, ces énonciations individuelles sont tronquées ici de leur inscription

\footnotetext{
${ }^{35}$ La méthodologie est qualitative. Le corpus utilisé dans cette contribution est de dix entretiens compréhensifs, d'une durée moyenne de $2 \mathrm{~h} 30$, auprès de bibliothécaires en poste. Ce corpus a fait l'objet d'une analyse de contenu thématique.

${ }^{36}$ Nous restons dans le registre du discours, c'est-à-dire de l'expression d'éléments ressentis et reconstruits des postures managériales que nos interlocuteurs adoptent ou dont ils font l'objet, ponctuant leurs propos de faits concrets «exemplaires». Ils font également référence à des réflexions professionnelles sur le management et sur le positionnement du bibliothécaire, créant ainsi un discours composite entre introspection et témoignage d'une représentation plus « atemporelle », et largement discutée, de l'activité et du rôle de cadre intermédiaire au sein de la profession.
} 
Dossier : Pour une approche communicationnelle de l'individu au travail

dans un groupe professionnel en lutte pour sa pleine reconnaissance. En effet, cette dernière est aujourd'hui mise en question par des décisions politiques, et objet de débats au sein de la profession ${ }^{37}$. Néanmoins transparaissent dans les paroles recueillies à la fois la diversité des situations, la fierté et l'intérêt pour ce métier de bibliothécaire et le malaise à l'origine de ces mouvements.

\section{Bibliothécaire et encadrement intermédiaire : un clair-obscur}

Encadrer. Un métier impossible? interroge Frederik Mispelblom Beyer (2006), en décrivant combien cette position d'encadrement est le lieu d'un «ferraillage» (ibid: 38 ) avec la situation, parfois de la navigation à vue et pourtant un entre-deux qui doit produire du sens, qui doit «tenter d'unifier les orientations de travail » en étant soumis «aux pressions du haut et du bas » (Mispelblom Beyer, 2006: 153 et al.). Position qui, dans le cas des personnels d'encadrement des bibliothèques (catégorie $\mathrm{A}$ de la fonction publique), est partagée par les conservateurs et les bibliothécaires, les seconds étant sous l'autorité hiérarchique des premiers. Or la ligne de partage des responsabilités est parfois ténue dans la réalité : des bibliothécaires peuvent diriger des établissements dans les collectivités territoriales (souvent de taille petite ou moyenne) ou être responsables de services (souvent les services au public) dans les bibliothèques universitaires... à salaire moindre. Plusieurs personnes interviewées dans notre étude se sont ainsi trouvées à certaines périodes en position de direction par intérim ou à assurer les fonctions d'un conservateur (de manière plus ou moins permanente).

À reprendre les activités, responsabilités et parcours des différents bibliothécaires interviewés, on aboutit ainsi plus à une mosaïque qu'à une vision uniforme de l'encadrement intermédiaire, supposée au départ de l'enquête. Saisie par le statut et non par la fonction, l'entrée ouvre sur des situations d'encadrement multiples, selon que l'on est intégré à l'équipe de direction dans tel établissement ou que l'on fait partie de l'encadrement de proximité dans tel autre... ou encore que l'on est investi de missions transversales, et d'une responsabilité d'équipe dont les membres émargent hiérarchiquement à d'autres services. On peut toutefois dégager des propos recueillis quelques traits communs et récurrents sur la manière dont l'activité d'encadrement est perçue. Quelle que soit sa position, le bibliothécaire se (re)présente le plus souvent comme «un relais», comme «un

\footnotetext{
${ }^{37}$ Voir par exemple à ce sujet des prises de position parues sur le blog du BBF (Bulletin des Bibliothèques de France).
} 
pivot » entre le directeur de l'établissement et l'équipe. Dégagé des responsabilités de la direction de l'établissement, le bibliothécaire est celui qui se veut et est accessible (à l'inverse du directeur ou d'autres $\mathrm{N}+1$ ), celui qui gère les questions du quotidien du travail. Être au plus près de son équipe... dans bien des cas se traduit aussi physiquement : le bibliothécaire travaille souvent dans la même zone géographique que son équipe. Les personnes interviewées témoignent également de la difficulté à tenir cette position : «faire corps » avec la hiérarchie, comme il a obligation de le faire, tout en étant directement en relation avec une équipe dont il peut comprendre certaines oppositions; relayer des directives, lorsqu'une part de l'information qui permettrait de leur donner sens - ou, pour reprendre l'un de nos interlocuteurs, de les «traduire en termes opérationnels au niveau de l'équipe »- ne lui a pas été fournie. Enfin la fonction de motivation des membres de l'équipe est fortement soulignée.

Rien de bien nouveau on le voit : ces traits ne sont pas spécifiques à la situation d'encadrement en bibliothèque. On les retrouverait vraisemblablement dans la plupart des représentations que les cadres se font de leur travail et de leur position dans l'organisation. De même les interviews se réfèrent à des styles de management qui, même s'ils ne sont pas tous participatifs, accordent une part importante à la subjectivité en mettant en avant, parfois de manière contradictoire voire contestable, les notions de compétence ou d'engagement des personnes dans le travail; thèmes omniprésents dans la pensée managériale actuelle (Brunel, 2008 ; Floris, 2000). Pourtant, combinés au fait qu'ils s'appuient sur une valeur forte du métier et s'inscrivent dans des contraintes liées à la fonction publique, ces traits donnent un éclairage particulier à une problématique de la reconnaissance.

\section{La reconnaissance : constat et gratitude}

Il y a une certaine difficulté à parler de la reconnaissance et de ses manifestations: c'est ce que l'on peut constater au travers des entretiens réalisées. Demandant un double regard réflexif sur soi (comme $\mathrm{N}+1$ et comme $\mathrm{N}-1$ ) l'exercice fait du coup surgir des décrochements, des différences de traitement. Les deux manières d'appréhender cette notion (Dejours, $2005: 122$ ) permettent d'avancer une première interprétation.

Pour cet auteur, la reconnaissance prend deux sens. D'une part celui de constat «c'est-à-dire reconnaissance de la réalité que constitue la contribution du sujet à l'organisation du travail ». Entrent dans cette catégorie les éléments de nos interviews qui portent sur l'activité réelle, sur le travail effectué, bien ou mal, et dont les personnes diront 
Dossier : Pour une approche communicationnelle de l'individu au travail

qu'il est important qu'il soit vu et fasse l'objet d'une parole. Et là, en tant qu'encadrant, le fait de remarquer de manière positive le travail réalisé donne aussi de la marge pour s'autoriser à porter un jugement négatif lorsque le travail n'est pas (ou mal) fait. Ce qui permet à la fois de préserver les faces (Goffman, 2003: 9 et 15) de chacun et le climat social de l'équipe dont le bibliothécaire a la responsabilité.

D'autre part, en terme de "gratitude pour l'apport des travailleurs à l'organisation du travail » (Dejours, 2005 : 122) : de larges parts des entretiens montrent que c'est à cette dimension marquée positivement qu'est associée le plus souvent et spontanément le mot de reconnaissance. Elle sera toutefois reçue de manière plus ambivalente, selon qu'il s'agit de soi, ou des membres de son équipe. Il sera également souvent précisé que les directions sont le plus souvent silencieuses... et que cela dépend de la personnalité du directeur, reléguant donc ce type d'échange à la sphère subjective.

\section{Visibilité et reconnaissance}

«Seen but unnoticed ${ }^{\mathbf{3 8}}$ pour reprendre à notre compte une formule d'Harold Garfinkel (2002: 36), le travail effectif au quotidien ne fera pas l'objet d'une grande attention s'il est bien fait. S'ils s'accordent parfois pour dire que, dans leur organisation, c'est le travail réalisé qui est l'objet de reconnaissance (plus que les individus ou les qualifications), tous nos interlocuteurs montrent, au travers des exemples donnés, que ce travail réalisé est toujours celui réalisé «en plus » de celui prescrit. Sinon, le travail au quotidien ne se fait remarquer que lorsqu'il y a problème.

Pour être reconnue, l'activité doit-elle passer par une mise en scène ? En d'autres termes, se faire reconnaître c'est-à-dire passer par un travail de mise en visibilité que l'individu doit réaliser (certaines personnes interviewées ont utilisé l'expression de «savoir se vendre »). Ou doit-elle procéder de quelque chose de «naturel», «transpirer» de l'activité comme le soulignait un autre de nos interlocuteurs. De son seul fait, l'activité serait remarquée, faisant ainsi partie d'une sorte de contrat psychologique entre l'individu et l'organisation (Delobre, Herrbach, Lacaze et al., 2005), où les notions de justice, de juste rétribution ou encore de don et contre-don sont à l'œuvre?

\footnotetext{
${ }^{38}$ Perçu mais non remarqué
} 


\section{Faire reconnaître son travail}

L'attente d'une gratitude des N+1 vis-à-vis de soi ne sera pas explicitement exprimée dans les interviews... mais on peut la deviner lorsque les interlocuteurs parlent de tel travail réalisé, tel surcroît d'énergie pour mener à bien une activité, qui n'ont pas été mentionnés par leur hiérarchie. La demande latente n'est pas celle d'une démonstration publique, mais au minimum celle d'une parole échangée dans le cadre d'une relation informelle, qui vient renforcer le cadre de l'estime de soi défini par Axel Honneth (2008).

À entendre nos interlocuteurs, cette reconnaissance du travail fait peu l'objet d'une mise en scène. Toutefois elle est issue d'un travail de construction : la reconnaissance prend appui sur le fait que l'on a pris des initiatives, que l'on a «donné de la couleur» à son poste, et que ces éléments singuliers puissent perdurer dans le temps «officiellement $»$. Deux notions sont alors convoquées : la confiance accordée et de manière très liée, l'autonomie... car, pour nos interlocuteurs, au-delà des paroles c'est cela qui rend compte de la reconnaissance, qui la marque. Encore faut-il qu'elle soit malgré tout inscrite dans des objets (organigramme, fiche de poste, fiche d'évaluation, inscription à la liste des «promouvables », notation) : autant d'éléments qui rendent visibles la contribution à l'organisation et la «considération» des $\mathrm{N}+1$ en retour. Autant d'éléments qui légitiment à leur tour la position du bibliothécaire comme encadrant.

De manière surprenante, car semblant être une ressource associée à la fonction d'encadrement, le rôle de l'information a souvent été évoqué dans ce processus de reconnaissance. Etre informé est ressenti comme une marque de reconnaissance de sa fonction. En permettant de donner sens à l'application des directives auprès de son équipe, d'apporter des réponses, elle donne les moyens au bibliothécaire de remplir le rôle difficile d'interface, pris, comme évoqué précédemment, entre pression du haut et pression du bas. En lui permettant de faire une traduction, cette ressource informationnelle le qualifie dans un rôle autre que celui de la simple courroie de transmission... ce qui ne rend pas pour autant la position plus confortable. Le $\mathrm{N}+1$ ne perçoit pas toujours toute la complexité de cette activité de traduction, ou du moins ne la prend-t-il pas toujours en compte. Ne pas être mis au courant, ne pas être associé, est alors ressenti comme un déni de reconnaissance de cette position d'interface.

Être à l'interface, c'est aussi voir son positionnement reconnu par son équipe. Tous nos interlocuteurs ont insisté sur ce retour. Être vu 
Dossier : Pour une approche communicationnelle de l'individu au travail

comme référents parce qu'on viendra les solliciter pour tel ou tel problème, particulièrement si ce n'est pas inscrit dans leurs fonctions, est particulièrement gratifiant. Il s'agit alors d'une reconnaissance «en actes», au cœur de laquelle compétences relationnelles ou expertises «techniques» sont mises en avant et réaffirmées. Elle contribue à l'estime de soi qui repose pour Axel Honneth sur la «conviction de la fonction sociale de son activité » (Renault, 2006 : 36). On peut toutefois rejoindre Alain Caillé (2007: 196) lorsqu'il s'interroge sur les rapports ou combinaisons que peuvent prendre entre eux les trois principes de reconnaissance distingués par Axel Honneth (2007), à savoir la sphère de l'intimité (confiance en soi, amour), la sphère juridique (respect de soi) et la sphère de la solidarité (estime de soi). Ramenés aux paroles de nos interlocuteurs, il semblerait que ces trois registres sont tour à tour convoqués et le plus souvent profondément entremêlés pour que se construise ce sentiment de reconnaissance.

\section{Reconnaître celui des autres}

Les bibliothécaires, en tant qu'encadrants, se perçoivent comme attentifs à la fois à l'activité des membres de leurs équipes et aux individus eux mêmes. Proche de leurs collègues ${ }^{39}$ ils prêteront attention à la charge de travail, qui à leurs yeux passe souvent pour invisible au niveau de la direction. Opérant sur le registre de la mise en visibilité et de la mise en valeur, ils n'omettront pas alors de remercier, de «congratuler», pour reprendre le mot d'un interlocuteur, individuellement et collectivement ou encore de distinguer une réalisation ou une initiative en la relayant parfois directement au $\mathrm{N}+1$. Cette proximité les rend aussi sensibles à l'engagement des individus... qui demeure souvent largement invisible pour les $\mathrm{N}+1$, surtout si ces individus sont affectés à des activités de routine ou à des services qui gèrent l'activité normale d'une bibliothèque. S'il est convenu de dire que tout le monde contribue à la qualité du service, particulièrement dans un métier qui met fortement en valeur le service rendu au public, il reste que cette contribution peut rester parfois singulièrement inaperçue.

\footnotetext{
${ }^{39}$ Le terme de subordonné ne convient pas complètement dans les cas où nos interlocuteurs étaient responsables d'équipes transversales. En effet dans ces cas-là, les membres de ces équipes restaient sous l'autorité de leurs chefs de service respectifs directs. Pour donner un exemple, le responsable de l'équipe transversale n'intervenait pas dans l'évaluation des membres de «son» équipe.
} 
Cette reconnaissance passe essentiellement par la communication : il s'agit de la parole au quotidien, le plus souvent individuellement, parfois sous forme collective lors des réunions, plus rarement par écrit (ou alors sous forme de courriel... mais parce qu'on n'a pas réussi à le faire de vive voix). Faite le plus souvent dans l'immédiateté, dans un cadre informel, la reconnaissance s'inscrit aussi dans une continuité qui, nous l'avons dit, permet de reconnaître l'activité de l'autre, mais aussi ses manquements, mêlant ainsi constat et gratitude.

L'entretien d'évaluation et ses documents sont également mentionnés, mais jamais immédiatement car ce sont des espaces de reconnaissance ambigus. Ils participent d'une idéologie contestée dans la fonction publique (Bérard, 2008). En même temps, l'idée d'un espace de discussion ainsi aménagé pour mettre en évidence une partie de l'activité (celle liée aux objectifs) est également ressentie par certains de nos interlocuteurs comme une avancée. Elle est alors comparée à la notation où n'existait aucune possibilité d'échanges, ce que souligne également Raymond Bérard (2008). Cette ambiguïté d'un espace reste, au-delà du dialogue et d'une possible reconnaissance, avant tout celle du jugement (Dupont, Dutrel, 2006 : 126). D'où l'insistance de passer par l'oral, de rectifier au plus vite les choses afin de ne pas avoir à stigmatiser une personne. D'où aussi cette attention à être « honnête » pour reprendre le terme de l'un de nos interlocuteurs, à dire et ce qui va et ce qui ne va pas, directement à la personne concernée.

Enfin, dans l'un des cas, la mise en visibilité de l'activité est instrumentée par des statistiques : pour une opération fastidieuse et longue d'inventaire, des comptages permettront, lors des réunions plénières, de rendre compte du travail en cours. Ce procédé, souvent utilisé à des fins de compétition entre individus dans le secteur commercial est présenté ici comme marque de reconnaissance du travail en train de se faire, permettant de ne pas passer sous silence une activité nécessaire mais non spectaculaire.

Un terme revient en force pour ces cadres : celui de la motivation. Les contextes ne laissent que peu de marges de manœuvre. Le recours aux primes est limité, de même que les promotions dont la mise en œuvre est soumise à des procédures strictes, dans le cadre défini par le statut, en particulier pour la fonction publique d'Etat. La situation est encore plus délicate à gérer lorsque les membres de l'équipe sont des contractuels. On peut alors comprendre l'importance donnée à ce qui peut donner du sens à l'activité en informant et en communiquant d'une part et en se tournant vers des formes de rétribution 
Dossier : Pour une approche communicationnelle de l'individu au travail

immatérielles d'autre part. Le bibliothécaire recourt alors aux possibilités de formation et aux tâches complémentaires enrichissantes $^{40}$, perçues comme autant de moyens de reconnaissance des membres de l'équipe. Il s'agit alors de repérer des compétences, de les mettre en valeur en proposant telle ou telle mission, sachant que ces propositions, le plus souvent sur la base du volontariat ou issues d'initiatives, sont toujours frappées d'ambivalence, pouvant prendre les figures d'un plaisir au travail, d'un investissement dans une trajectoire professionnelle ou d'un sentiment d'exploitation.

\section{En guise de conclusion}

Cet article ne peut rendre compte de la multiplicité des éléments recueillis auprès de nos interlocuteurs sur ce que pouvait signifier en actes ou en paroles la reconnaissance dans le cadre de leur travail. C'est à partir d'un angle particulier ${ }^{41}$, celui de la visibilité ou mise en visibilité, que nous nous sommes attachée à comprendre ce qui pour les bibliothécaires peut porter les marques de la reconnaissance de leur travail mais aussi de celui de leurs équipes, dans le cadre contraignant de l'organisation et des interrelations humaines. Pour ce faire, certains outils (fiche de poste, etc.) sont parfois utilisés. Les quelques dispositifs mentionnés par nos interlocuteurs et sur lesquels ils peuvent prendre appui sont ambigus. S'ils peuvent être considérés comme instruments d'assujettissement, ils peuvent également être perçus comme support de reconnaissance et permettre de contribuer à l'estime de soi, en montrant l'utilité ou la réalité d'une contribution dans l'interaction.

Informer et communiquer se trouvent être des vecteurs privilégiés pour marquer la considération et la reconnaissance. Ils ne sont pas pour autant considérés ni comme allant de soi, ni comme suffisants. Nos interlocuteurs montrent combien les organisations restent silencieuses au quotidien sur les contributions des uns et des autres, du moins tant qu'il n'y a pas de visibilité extérieure (à la bibliothèque) de l'activité. Pour autant, «dire » ne suffit pas. C'est en effet pour nos interlocuteurs le minimum attendu, d'où leur frustration lorsque la juste rétribution - qu'elle ait pour manifestation le maintien dans une

\footnotetext{
${ }^{40}$ Enrichissantes non pas « en soi », mais aux yeux de nos interlocuteurs.

${ }^{41}$ D'autres questions, qui renvoient à d'autres dimensions que celles de la visibilité, comme les figures du mépris, le caractère instrumental que pourrait prendre la reconnaissance comme dispositif managérial, la question d'un modèle qui pourrait se dégager de la confrontation «soi comme encadrant, soi comme encadré » n'ont pas été adressées ici
} 
fonction «intéressante », la prime, la notation ou la promotion - ne suit pas les mots de la reconnaissance.

\section{Bibliographie}

BERARD R. L'évaluation des personnels dans les bibliothèques d'enseignement supérieur: espoirs et doutes. in: $B B F, 2008$, t. 53, n³, pp. 63-74

BRUNEL V. Pratiques réflexives et régulation organisationnelle. in : Communication \& Organisation, 2005, n ${ }^{\circ} 28$, pp. 17-32

CAILlE A. Reconnaissance et sociologie. in: La quête de reconnaissance. Nouveau phénomène social total. Paris: La découverte, 2007, pp. 185-208

CHARASSE D. L'usine, l'écriture et la place. in : Genèses, 1992, 7, pp. 63-93

DEJOURS C. Souffrance en France. La banalisation de l'injustice sociale. Paris : Seuil, 2006, 225 p.

DEJOURS C. L'évaluation du travail à l'épreuve du réel, critique des fondements de l'évaluation. Paris : INRA, 2003, 82 p.

DEJOURS C. Travail, usure mentale. Paris : Bayard, 2005, 280 p.

DELOBRE N., HERRBACH, O., LACAZE, D. et al. Comportement organisationnel. Vol. 1. Bruxelles : De Boeck, 2005, 407 p.

DUPONT O., DUTREL S. Entretien annuel d'évaluation. Le document, un objet intermédiateur. in: Sciences de la société, 2006, $\mathrm{n}^{\circ} 68$, pp. 111-131

FLORIS B. La gestion symbolique entre ingénierie et manipulation. in : Sciences de la société, 2000, nº50-51, pp.173-195

GARFINKEL H. Studies in ethnomethodology. Cambridge: Polity Press, 2002

GOFFMAN E. Les rites d'interaction. Paris : Éd. de Minuit, 2003, $230 \mathrm{p}$.

HONNETH A. La lutte pour la reconnaissance. Paris : Éd. du Cerf, coll. Passages, 2008, 232 p.

HONNETH A. Invisibilité : sur l'épistémologie de la reconnaissance. in: Réseaux, $\mathrm{n}^{\circ}$ 129-130, FT R\&D/Lavoisier, 2005, pp. 40-57 
Dossier : Pour une approche communicationnelle de l'individu au travail

LARROCHE V., Reconnaissance et médiation. in : Actes du $16^{\text {ème }}$ Congrès de la SFSIC, Compiègne, 11-13 Juin 2008, disponible sur : http://www.sfsic.org/congres_2008/spip.php?article25

LARROCHE V., PEYRELONG M.F., LEPINE V., LAROUX M.N., Reconnaissance dans l'organisation: la représentation des bibliothécaires. in: Actes du colloque I3M, Les dispositifs de Médiation organisationnelle, technologique et symbolique dans la communication des organisations », Nice 4-5 Décembre 2008, pp. 93101

MISPELBLOM BEYER F. Encadrer. Un métier impossible? Paris : Armand Colin, 2006, 301 p. (Sociétales)

RENAULT E. La reconnaissance au cœur du social. in: Sciences humaines, $2006, \mathrm{n}^{\circ} 172$, pp. 34-37

THEVENOT L. Les investissements de forme. in: Conventions économiques. Paris, PUF, 1986, pp. 21-71 (Cahiers du centre d'étude de l'emploi)

VOIROL O. Visibilité et invisibilité : une introduction. in : Réseaux, 2005, n $129-130$, pp. 9-36

VOIROL O. Les luttes pour la visibilité. Esquisse d'une problématique. in : Réseaux, 2005, n 129-130, pp. 89-121 